Alexandre Leite da Silva

Universidade Federal do ABC (Brésil)

Maisa Helena Altarugio

Universidade Federal do $A B C$ (Brésil)

\section{Les conditions favorables à la réussite d'un stage selon les stagiaires en formation au Brésil}

\author{
The favorable conditions for the success of an internship \\ according to trainees in training in Brazil
}

doi: $10.18162 / f p .2021 .606$

\section{ésumé}

À partir du contexte brésilien des stages supervisés, la présente recherche analyse comment

l'école, le superviseur et l'enseignant associé promeuvent des expériences de stage réussies.

Dans cette recherche qualitative, vingt-trois stagiaires d'une université publique ont répondu à un questionnaire concernant leurs expériences de stage. Les résultats montrent que les expériences

réussies sont celles où les stagiaires se sentent sûrs, bien accueillis et encouragés, ce qui démontre la nécessité de perfectionner les compétences des agents de formation et de valoriser les conditions déjà existantes. L'articulation entre l'université et l'école est l'un des défis les plus importants dans le domaine des stages.

\section{Mots-clés}

Stages supervisés, enseignant associé, superviseur, expériences de stages.

Abstract

In the Brazilian context of supervised internships, the present study investigates how schools and teacher trainers have to provided successful experiences for interns. In this qualitative research, twenty-three interns from Brazilian public university have answered a questionnaire about their internship experiences. The results point out that the successful experiences are those where the interns can feel safe, welcome and encouraged, demonstrating the need to upgrade the skills of training agents and to enhance existing conditions. The articulation between university and school appears to be one of the main challenges of the internships.

Keywords

Supervised internships, teacher trainers, intern experiences.

\section{Introduction}

Au Brésil, les stages supervisés sont une activité obligatoire dans la formation des enseignants du primaire et du secondaire, prévue et soutenue par la Loi de Directives et de Bases de l'Éducation Nationale (LDBEN) dans le cadre du Ministère de l'Éducation (Brésil, 1996) et visent à offrir aux futurs enseignants une connaissance de la situation réelle de travail. Ces stages ont une durée minimale de 400 heures de stage et doivent être réalisés à partir de la mi-parcours du cours de Licence. Conformément à la législation brésilienne, la licence n'est obtenue qu'après la réussite du stage (Brésil, 2001a, 2001b).

Selon des chercheurs du domaine des stages supervisés, des expériences réussies dans ce moment initial de formation doivent assurer aux stagiaires l'occasion d'articuler savoirs théoriques et savoirs pratiques (Pimenta et Lima, 2004; Caron et Portelance, 2017, Portelance et Van Nieuwenhoven, 2010) et aussi constituer et stimuler des moments de réflexion sur la profession d'enseignant (Chaliès et Durand, 2000); rendre possible le développement de compétences professionnelles et de savoir-faire en mobilisant diverses ressources (Gervais, 2005; Le Boterf, 2002; Altet, 2000); être soutenus et supervisés par des enseignants capables et expérimentés (Lamy, 2003); offrir une ambiance accueillante, de sécurité et de confiance aux acteurs impliqués (Correa Molina, 2008; Gervais et Desrosiers, 2005). En outre, l'obtention de résultats positifs aux stages supervisés exigerait un travail bien articulé et intégré entre l'université et l'école (Cyrino et Souza Neto, 2014; Souza Neto, Sarti et Benites, 2016; Gervais, 2013). 
Compte tenu des études mentionnées ci-dessus qui discutent les éléments qui peuvent influencer positivement les stages au Brésil, pays où les inégalités et les particularités dans les stages persistent, le présent travail a donc pour but d'analyser, du point de vue des stagiaires, soumis à différents agents de formation, comme l'école, le superviseur et l'enseignant associé, les conditions qui leur permettent d'avoir des expériences satisfaisantes et réussies dans leurs stages. En outre, nous cherchons à dévoiler et à discuter les défis avec lesquels les institutions formatrices d'enseignants des écoles secondaires doivent composer pour améliorer la qualité des stages et la formation des futurs enseignants.

Ensuite, on passera à la problématisation du rôle des professionnels de l'université et de l'école dans la gestion des stages à partir d'un bref panorama national en apportant quelques références francophones pour aider à comprendre le contexte brésilien.

\section{Les professionnels de l'université et de l'école dans la gestion des stages}

Pour plusieurs étudiants, le stage joue un rôle décisif dans le choix de la carrière d'enseignant. Ce choix est probablement influencé par le parcours et les expériences personnelles des stagiaires. Les expériences réussies dépendent d'un programme de stage ayant une base théorico-scientifique solide, capable d'offrir au stagiaire l'occasion de développer ses compétences (Pimenta et Lima, 2004;Rodrigues, 2013; Caron et Portelance, 2017); qui encourage l'autonomie (Altarugio et Souza Neto, 2018; Milanesi, 2012) et qui soit supervisé par des professionnels engagés et attentifs aux problèmes qui surgissent pendant le stage (Martins, 2009).

Les directives légales pour la formation des enseignants au Brésil font des recommandations concernant les qualifications que doit avoir un professionnel responsable pour la gestion des stages dans les universités et soulignent qu'il faut que ce soit « un professionnel reconnu et expérimenté dans son cadre institutionnel de travail » (Ministério da Educação, 2001b, p.8). D'autre côté, les directives ne formulent pas de recommandations spécifiques quant aux professionnels chargés d'accueillir les stagiaires dans les écoles où se déroulent les stages, ce qui peut nuire à la qualité de la formation des futurs enseignants.

Selon Altarugio et Souza Neto (2018), il est rare que les universités et les écoles qui recevront les stagiaires trouvent des professionnels reconnus et expérimentés dans le domaine des stages. Au Brésil, des enseignants associés et superviseurs débutants ou peu expérimentés finissent souvent par s'occuper de ce travail et construisent leur rôle à partir de leurs savoirs de formation, issus des programmes et des outils dont ils se servent au travail, ou bien encore les savoirs issus de leur expérience professionnelle (Tardif, 2010). En plus de ces savoirs, les études de Rozelle et Wilson (2012) démontrent l'influence des valeurs et des croyances des superviseurs sur les pratiques des stagiaires.

Cependant, les savoirs professionnels ne sont pas suffisants pour qu'un professionnel puisse s'occuper des stages. Selon Altet, Perrenoud et Paquay (2003), on doit tenir compte des spécificités et des différences qu'il y a entre un enseignant et un formateur d'enseignants. Pour Lamy (2003), par exemple, il faut construire un nouveau répertoire de connaissances capable d'offrir des conditions pour gérer cette période du développement professionnel. La recherche de Brau-Antony et Mieusset (2013) ajoute au manque d'une formation spécifique pour les superviseurs le fait que ceux-ci se sentent seuls et abandonnés à leur sort ainsi que dépourvus de moyens pour régler les problèmes auxquels ils doivent faire face.

2 - Formation et profession 29(3), 2021 
Dans ce contexte, le rôle de l'enseignant associé est aussi important que celui du superviseur. Selon Mazieiro et Carvalho (2012), il est souhaitable que l'enseignant associé ne se limite pas à observer, mais qu'il soit actif in situ et participe aux actions des stagiaires sous sa tutelle. Linhares, Irineu, Silva, Figueiredo et Sousa (2014) soulignent l'importance de la participation collaborative des deux au processus d'enseignement et d'apprentissage et également dans leur action dans l'environnement scolaire, avec la possibilité d'intervention dans des situations-problème réelles dans la salle de classe.

Plusieurs études réalisées par des chercheurs du Québec attestent de l'importance des contributions des enseignants associés pour le développement professionnel des stagiaires, surtout en ce qui concerne la formation préalable pour l'exercice de ce travail (Gervais, 2013; Gervais et Lepage, 2000; Desbiens, Borges et Spallanzani, 2009). Portelance, Gervais, Lessard et Beaulieu (2008), par exemple, proposent une liste des compétences que les enseignants associés devraient perfectionner : encadrer le stagiaire avec professionnalisme (observation rigoureuse, rétroaction constructive et évaluation continue et fondée); collaborer avec les différents intervenants; soutenir la construction de l'identité professionnelle du stagiaire; agir de façon éthique; interagir avec respect et de manière à établir une relation de confiance avec le stagiaire.

Au Brésil, les directives officielles ne prévoient pas la préparation des enseignants associés pour accueillir les stagiaires, mais parfois les universités offrent aux enseignants associés l'occasion de planifier un projet de stage (Ministério da Educação 2001a). Vu la superficialité des textes des directives officielles, en général les universités sont responsables de déterminer les critères de formation et d'évaluation des stagiaires.

Puisque les universités et les superviseurs sont libres de définir leurs programmes de stage, il y a une énorme variété de projets et d'actions dans ce domaine, ce qui cause une grande inégalité dans l'apprentissage des stagiaires et, par conséquent, dans la qualité de la formation des enseignants. Souvent, les superviseurs ne connaissent pas le quotidien des écoles et n'ont pas d'expérience en enseignement scolaire. Selon quelques chercheurs brésiliens (Lüdke, 2009; Martins, 2009; Aroeira, 2015), une collaboration entre l'université et l'école serait souhaitable lors de l'évaluation des stagiaires.

Martins (2009) souligne qu'il est impossible pour les superviseurs de faire l'accompagnement in situ des stagiaires quand les universités reçoivent des étudiants de villes voisines. Selon Scalabrin et Molinari (2013), cela se passe, car les stagiaires choisissent les écoles où ils ont fait leurs études primaires et secondaires, parce qu'ils se sentent plus à l'aise dans des ambiances déjà connues, avec lesquelles ils sont déjà familiarisés. Selon Martins (2009), ces écoles ont souvent des conditions matérielles et humaines précaires : mobiliers abîmés, ampoules grillées, absence de laboratoires de science et d'informatique et parfois même de bibliothèques, et un nombre d'enseignants insuffisant dans les écoles pour accompagner les stagiaires. Toutefois, il est parfois possible d'y trouver des enseignants capables d'offrir aux stagiaires des expériences enrichissantes grâce à leur soutien dans les activités et aux bonnes relations qu'ils entretenaient avec les étudiants.

Malheureusement, l'école est encore envisagée par les stagiaires comme un lieu où ils vont appliquer les connaissances acquises à l'université et non pas comme un espace de formation (Behrend, Cousin et Schmidt, 2019). Selon Pimenta et Lima (2004), le stage doit être compris comme un « champ de connaissance » et de production de savoir, un « espace de réflexion sur la construction et le renforcement de l'identité » des enseignants et non pas comme une « activité technique ». 
Malgré les difficultés et les défis auxquels doivent faire face les stages au Brésil, serait-il possible d'assurer aux étudiants des expériences réussies dans leurs stages? Notre but est d'identifier les conditions citées dans les témoignages des stagiaires qui ont rendu possible la réalisation d'expériences satisfaisantes, ayant pour interlocuteurs les principaux acteurs dans leur formation, comme les professeurs, les superviseurs et les écoles où ils ont fait leurs stages. Des expériences jugées partiellement satisfaisantes ou insatisfaisantes ont fourni des indices au sujet des défis qu'il faut envisager dans ce domaine.

\section{Méthodologie}

\section{Sur le lieu de la recherche et le profil des sujets}

La recherche a été menée dans une université publique de São Paulo, au Brésil, qui offre des programmes de formation d'enseignants en chimie, en physique, en mathématiques, en biologie et en philosophie depuis 2009. Chacun de ces cours possède une durée minimale de 3200 heures, soit 4 ans de formation, y compris les heures de stage, et les étudiants commencent leurs stages supervisés obligatoires à partir de la mi-parcours du programme. Les 400 heures de stage sont divisées en 5 modules de 80 heures destinées à des rendez-vous du professeur superviseur avec son groupe de stagiaires, aux activités dans les écoles, aux études personnelles et à l'élaboration de rapports. Les stages se déroulent en même temps que les autres contenus du cours.

Une particularité des stages de cette université est l'existence de formes spécifiques concernant les projets développés par les stagiaires (méthodologies, stratégies, évaluation, rapports) qui varient selon chaque cours et chaque professeur universitaire chargé de la supervision. Malgré l'existence d'un Règlement Interne et d'un Manuel de Stages Supervisés qui établissent les normes pour les superviseurs et les stagiaires, la liberté pédagogique est assurée aux superviseurs. Il faut dire que, dans cette université, chaque superviseur est chargé d'accompagner des groupes de 2 à 15 stagiaires et doit aussi de s'occuper des tâches administratives et académiques.

Dans cette université, il n'y a aucune sorte d'accord formel entre l'institution formatrice et les écoles qui recevront les étudiants. Malgré la recommandation de l'université suggérant que la majorité des heures de stage soit réalisée dans des écoles publiques, il est possible que les stagiaires décident de faire leurs stages dans une école privée ou bien dans d'autres espaces d'éducation non formelle.

Un questionnaire a été envoyé à un réseau social créé exclusivement pour les stagiaires des cinq programmes de licence de l'université. Seuls ceux qui ont réalisé leurs stages exclusivement dans des écoles publiques de la région où se trouve 1'université ont été invités à y participer. Des 43 stagiaires qui ont reçu le questionnaire, il y en avait 38 qui répondaient à cette exigence; nous avons obtenu 23 réponses. Pour assurer l'anonymat, les sujets sont identifiés par les sigles E1 à E23 aléatoirement.

Des 23 répondants de cette recherche, 11 sont étudiants en chimie, 5 étudiants en mathématiques, 4 étudiants en physique, 2 étudiants en biologie et 1 étudiant en philosophie. Tous ont choisi de réaliser leurs stages exclusivement dans des écoles publiques situées près de l'université.

4 - Formation et profession 29(3), 2021 


\section{Sur la nature et les procédures de la recherche}

La présente étude est une recherche qualitative, puisque la base de données non métriques et descriptives tient compte du point de vue du répondant, quelle valorise son interprétation, sa réalité, son contexte ainsi que sa vision de monde de la manière la plus fidèle possible (Bodgan et Biklen, 1994).

La collecte de données a été réalisée au moyen d'un questionnaire ayant trois questions à choix multiples avec justifications et une question ouverte. Pour les trois premières questions à choix multiples, les stagiaires ont évalué leurs expériences pendant le dernier module de leurs stages, selon une échelle Likert de satisfaction avec trois options de réponse : "satisfait », " partiellement satisfait » et « insatisfait ». Chacune de ces questions a mesuré la satisfaction avec l'école, l'enseignant associé et le superviseur. Les réponses étaient suivies d'une justification.

La quatrième question, ouverte, avait pour but de récolter des témoignages des stagiaires sur leurs perceptions de l'articulation entre l'école, le superviseur et l'enseignant associé pour découvrir si cette articulation avait contribué à des expériences réussies. Les éléments les plus valorisés par les stagiaires dans leurs expériences satisfaisantes nous ont permis de mieux comprendre ce qui, à leur avis, contribue à la réussite de l'expérience et ont fourni des pistes pour améliorer ces expériences. Les questions seront présentées dans la section Résultats.

Les questionnaires de satisfaction avec des étudiants de l'enseignement supérieur font référence aux expériences subjectives de l'étudiant pendant son parcours et à leur perception des attentes et besoins satisfaits (Nico, 2000). L'évaluation que l'étudiant fait de ses résultats et de son expérience peut être influencée par l'ambiance institutionnelle (Elliot et Shin, 2010), par la qualité de la formation, par les relations avec les professeurs et les collègues, par sa perception du temps et de l'effort pour accomplir sa formation (Carmo et Polydoro, 2010). Les questionnaires de satisfaction offrent à l'institution l'occasion de connaître ses points forts et de perfectionner les programmes et les services offerts aux étudiants dans le but d'améliorer la satisfaction de l'étudiant (Schleich, Polydoro et Santos, 2006).

Le questionnaire à réponses ouvertes permet au participant de répondre en se servant de ses propres mots et d'émettre ses opinions (Marconi et Lakatos, 1990). Cette technique a pour but de connaître les croyances, les sentiments, les intérêts et les situations vécues par les stagiaires (Gil, 1999). Dans la présente recherche, les questions identiques ont assuré que tous les thèmes soient abordés par les répondants. Selon Malhotra (2006), malgré les avantages des sondages effectués par Internet, le nombre des réponses y est souvent très faible. Cela n'empêcherait pas l'analyse quantitative des données qui, dans notre cas spécifique, s'est limitée à indiquer les fréquences des réponses obtenues.

Les données collectées ont été analysées en vue d'interpréter les réponses données à chacune des questions par l'analyse de contenu (Bardin, 2008). Les réponses ont été regroupées selon les options établies par les instruments de collecte. Au début, l'exploration du matériel par la lecture fluctuante des réponses a rendu possible l'identification des unités d'analyse et, par conséquent, la division en catégories et sous-catégories d'analyse par fréquence des données (répétition de contenus communs à la majorité des répondants) et par pertinence implicite (thème important qui ne se répète pas dans les témoignages de tous les répondants, mais qui s'avère important pour l'étude). Toutes les étapes de l'analyse sont validées par les auteurs et sont basées sur les principaux référentiels théoriques s'inspirant de Le Boterf, Gervais, Correa Molina, Altarugio et Souza Neto. 


\section{Résultats}

Premièrement, nous analyserons seulement les expériences jugées satisfaisantes par les stagiaires par rapport à l'école, à l'enseignant associé et au superviseur. Deuxièmement, nous nous pencherons sur les défis qu'impliquent les stages supervisés au Brésil à partir des expériences jugées partiellement satisfaisantes ou insatisfaisantes par les étudiants.

\section{L'école}

Les 23 stagiaires qui ont participé à la recherche ont répondu à la question : « considérant votre dernière période de stage, attribuez à l'agent École le niveau de satisfaction avec les expériences vécues ». De ces 23 stagiaires, 19 répondants se déclarent satisfaits, 3 se disent partiellement satisfaits et 1 stagiaire se dit insatisfait. À partir des justifications des stagiaires, nous pouvons souligner deux aspects de l'école qui favorisent le déroulement du stage : l'accueil au stagiaire et l'occasion de vivre des situations pratiques.

Concernant l'accueil au stagiaire, les étudiants ont attribué l'accueil positif surtout aux gestionnaires de l'école (directeurs et coordinateurs). Les témoignages des stagiaires E7 et E9 en sont un exemple : « L'école nous a très bien reçus, nous a laissés libres d'observer les divers aspects structuraux et comportementaux de l'école» (E7); "les gestionnaires de l'école accueillaient toujours très bien les stagiaires et leur permettaient de s'intégrer au quotidien de l'école» (E9).

L'accueil positif du personnel de l'école est démontré par la liberté et la confiance accordées aux stagiaires et aussi par l'intégration de ces stagiaires au quotidien de l'école: "Je crois que l'école a été cruciale pour ma satisfaction pendant mon dernier stage, vu que la coordination, les professeurs et les élèves, tous étaient prévenants lors de toutes les activités proposées» (E22). La possibilité d'établir des liens semble être une des raisons de la réussite de l'expérience : "J'ai fait mon stage à l'école où j'ai fait mes études, alors je connaissais déjà quelques enseignants, l'enseignant associé et les employés. À mon avis cela a été important pour le déroulement de mon stage» (E3).

Sur l'occasion de vivre des situations pratiques dans l'environnement scolaire, les stagiaires ont souligné l'importance d'être en contact avec la réalité scolaire et d'apprendre à maîtriser les problèmes quotidiens en tant que futurs enseignants :

J'ai accompagné les enseignants qui préparaient des activités pour leurs groupes et qui participaient aux conseils de classe. Pour moi ces expériences ont été très importantes, la première parce que je n'avais aucune idée de comment le faire et la deuxième parce que je me suis mis du côté des enseignants et j’ai pu voir comment ça fonctionne (E19).

L'école peut devenir un environnement très enrichissant pour les stagiaires, pourvu qu'elle donne un accès sans réserve aux problèmes complexes du quotidien de l'école : "J'ai eu l'occasion d'accompagner un groupe d'élèves dont deux étaient hyperactifs et un autre avait des troubles de comportement. Ca a été très enrichissant de voir comment les enseignants, les autres élèves et l'école maîtrisaient la situation» (E19). Voici un exemple de la manière dont le contact direct avec l'expérience des stages peut apporter aux stagiaires un apprentissage décisif, capable de les aider à choisir la profession d'enseignant. 


\section{Le superviseur}

Concernant la contribution du superviseur, les 23 stagiaires qui ont participé à la recherche ont répondu à la question : «considérant votre dernière période de stage, attribuez à l'agent Superviseur le niveau de satisfaction avec les expériences vécues ». Vingt stagiaires déclarent avoir eu des expériences satisfaisantes et 3 stagiaires se disent partiellement satisfaits. Quand nous avons analysé les témoignages des stagiaires satisfaits, nous avons pu constater l'importance de ses qualités professionnelles, associées à la compétence du "savoir agir "; nous les avons classées en deux catégories: disponibilité et encouragement à la réflexion.

La disponibilité, selon les stagiaires, se reflète surtout dans les réponses aux doutes et dans l'attention portée aux difficultés des étudiants, comme le démontrent les témoignages retenus : «Le superviseur a commenté toutes les activités et était toujours disponible pour parler de mes difficultés» (E3); «le superviseur m'a accompagnéà l'école et a pris des notes sur mon travail, c'était très enrichissant, on ne voit pas ça très souvent » (E7); «le superviseur a été prévenant, ouvert aux doutes et aux suggestions » (E14). La disponibilité des superviseurs se restreint donc aux rendez-vous hebdomadaires, très prisés par les étudiants: "quand les groupes étaient petits, la professeure nous accompagnait de près » (E17).

L'encouragement à la réflexion, pour chaque stagiaire, demande un effort du superviseur pour diriger le regard de l'étudiant vers un aspect différent du stage. "Le superviseur m'a sensibilisé au sérieux et à la responsabilité du stage supervisé" (E1); "le superviseur a attiré l'attention des étudiants à des aspects auxquels on ne ferait pas attention seuls" (E7); "le superviseur nous encourage toujours et nous incite à la réflexion sur notre rôle de futurs enseignants" (E9); "il nous a appris à avoir une vision critique non seulement des pratiques pédagogiques, mais aussi des enjeux scolaires » (E20).-

Nous constatons que l'objet de la réflexion, selon les étudiants, semble dépasser l'aspect pédagogique. Quelques enseignants associés attirent l'attention des stagiaires sur le rôle professionnel et social de l'enseignant, étant donné qu'il réfléchit aux responsabilités de l'enseignant au-delà de la salle de cours.

\section{L'enseignant associé}

Les 23 stagiaires qui ont participé à la recherche ont répondu à la question : « considérant votre dernière période de stage, attribuez à l'agent Enseignant associé le niveau de satisfaction avec les expériences vécues ». Quinze d'entre eux sont satisfaits, 5 se déclarent partiellement satisfaits et 3 sont insatisfaits des contributions de cet agent de formation. Il faut tenir compte du fait que l'enseignant associé, si possible, est choisi par le stagiaire selon ses expériences préalables et sa relation avec cet enseignant ou celui qui est disponible à l'école dans les horaires convenables pour le stagiaire. Or, la réussite des expériences avec ces professionnels, selon les stagiaires, dépend plutôt de leur attitude par rapport aux stagiaires, c'est-à-dire : de la liberté d'action, de l'insertion dans le quotidien de l'école et de l'échange d'expériences.

La liberté d'action signifie, pour les stagiaires, la possibilité de choisir, de suggérer des activités et de discuter de leurs doutes sans s'abstenir de l'aide des enseignants associés : "le plus important dans cette expérience c'est l'enseignant associé qui a discuté avec moi sur les activités et m'a laissé à l'aise " (E3); "j'ai eu l'occasion de mettre en marche des activités à l'école avec le soutien de l'enseignante associée $»($ E10). 
La pratique d'insérer le stagiaire dans le quotidien de l'école est vraiment valorisée par les étudiants. Il semble important pour ces stagiaires de se sentir intégrés à l'environnement scolaire, y compris tous les espaces et activités où ils puissent observer le travail de l'enseignant associé : «l'enseignante a été extrêmement prérvenante, elle m'a intégré au quotidien de l'école " (E8); "j'ai trouvé très important d'accompagner le quotidien de l'enseignant, il m'a donné plein de conseils, m’a parlé un peu de quelques élèves et de la dynamique de l'école » (E5).

Les moments d'échange d'expériences et de savoirs font partie des attentes des stagiaires et suscitent aussi la réflexion : "en analysant le comportement de l'enseignant associé, j'ai pu apprendre comment me porter dans certaines situations " (E1); "j'ai pu voir différentes interprétations et solutions proposées par élèves pour les exercices et cela m'a fait questionner la nature de l'exercice et son évaluation "(E20).

\section{Les défis des stages supervisés : des aspects à surmonter}

Quand nous analysons les expériences jugées partiellement satisfaisantes et insatisfaisantes par rapport aux contributions des trois agents de formation, nous pouvons mieux comprendre les difficultés envisagées par les stagiaires.

La majorité des témoignages semble être motivée par des situations spécifiques; par conséquent, nous avons éprouvé des difficultés pour établir des catégories. Néanmoins, une question que nous considérons comme problématique et sérieuse, puisqu'elle est présente dans plusieurs témoignages, avec différentes nuances, est associée au (manque de) préparation préalable de l'école et surtout des enseignants associés. Les témoignages suivants confirment les problèmes signalés dans les recherches mondiales dont nous avons déjà parlé. Toutefois, ces témoignages dévoilent un peu la nature des défauts que les stagiaires ont observés pendant leurs stages : "j’ai eu l'impression que l'enseignant associé était un peu mal à l'aise avec ma présence en salle de classe" (E1); "l'enseignant associé ne savait pas comment m'aider, c'était difficile de négocier les activités »(E3); "le directeur de l'école demandait toujours des dossiers de la faculté. Je crois qu'il voulait rendre le processus plus difficile » (E5); " les enseignants associés sont fatigués de leur quotidien, ils n'ont pas vraiment fait l'accompagnement des stagiaires 》(E14).

Ces témoignages nous font supposer l'existence d'une certaine incompétence de quelques enseignants associés pour accompagner les stages, causée peut-être par les faiblesses, par le désintérêt et par la méconnaissance du processus et l'importance du stage, voire par des problèmes éthiques. Même s'ils sont rarement mentionnés, les gestionnaires semblent être les agents les moins concernés par les stagiaires.

L'articulation entre l'école, le superviseur et l'enseignant associé, d'après les résultats obtenus à partir de la question «considérant votre dernière période de stage, vous constatez l'articulation entre l'école, le superviseur et l'enseignant associé", sera considérée comme une catégorie d'analyse spécifique parmi les défis du stage. Plus de la moitié des stagiaires affirment que cette articulation n'existe pas, ne s'en rendent pas compte ou la croient insuffisante : "je n'ai vu aucune articulation » (E6); "non, il n'y a pas d'interaction entre eux [les agents]»(E23). D'autres témoignages reconnaissent l'importance de cette articulation: "c'est évident pour moi que l'école et l'université doivent travailler toujours ensemble » (E22). 
Il y a aussi des témoignages qui suscitent des hypothèses sur les raisons pour lesquelles l'articulation n'a pas eu lieu ou était insuffisante : "le stagiaire est le seul responsable pour le contact avec l'école et l'enseignant associé. Après être accepté à l'école, je n'ai discuté du stage qu'avec l'enseignant associé, l'école n’intervient pas » (E3); "le stagiaire est chargé de l'articulation et on dépend énormément du professeur de l'université » (E12). Rares ont été les témoignages constatant l'existence d'une véritable articulation et qui en ont donné des exemples: "le superviseur de l'université a contacté l'école, a informé le but du stage, sont venus à tous les rendez-vous, mais cela est une exception» (E21).

Dans tous ces témoignages, il semble que l'articulation est inhabituelle et qu'elle dépend de l'initiative, de la disponibilité ou des conditions des formateurs et de l'école où a lieu le stage. Il n'est donc jamais sûr que cette articulation se fera toujours.

\section{Discussions}

En général, les expériences des stagiaires démontrent l'importance des superviseurs, dont le travail a obtenu les taux de satisfaction les plus élévés : 20 répondants satisfaits, suivi des écoles, avec 19 répondants. Le travail des enseignants associés, en revanche, a obtenu les plus faibles taux de satisfaction : 15 répondants satisfaits. La présente recherche nous a permis de découvrir quelles conditions offertes par ces agents de formation sont perçues par les stagiaires comme décisives pour leurs expériences réussies et aussi quels sont les problèmes auxquels nous devons faire face pour obtenir de meilleurs résultats.

D'après nos interprétations et selon un ensemble d'indicatifs que nous avons présentés sur les conditions nécessaires pour obtenir des expériences réussies, les expériences de stages supervisés réussies sont celles où les stagiaires se sentent sûrs d'eux-mêmes et valorisés, c'est-à-dire, au cours desquelles ils peuvent compter sur le soutien des agents de formation. Autrement dit, ce sont ces sentiments qui permettent aux stagiaires de développer leurs compétences, leurs savoir-faire et leurs ressources personnelles (Le Boterf, 2002, Gervais, 2005; Altet, 2000). Ce sentiment de sécurité et cette valorisation dépendent aussi de l'environnement de l'école et de l'attitude des professionnels impliqués dans leur formation (Correa Molina, 2008). Les stagiaires veulent faire leurs stages dans des écoles qui les reconnaissent comme de futurs enseignants qui pourront bientôt faire partie de leur communauté; ils veulent donc bien connaître ces espaces, y interagir et maîtriser leurs problèmes réels (Linhares et al., 2014).

La recherche de ce sentiment de sécurité est associée à l'établissement de liens, visiblement nécessaires dans ce contexte (Correa Molina, 2008). Les expériences réussissent mieux quand les stagiaires et les enseignants sont plus proches, quand les stagiaires sont accompagnés par les superviseurs dans les écoles et quand il y a une relation de confiance entre l'école et l'université (Gervais et Desrosiers, 2005). Les stagiaires se sentent à l'aise pour exposer leurs idées et opinions quand le formateur possède des qualités personnelles (Le Boterf, 2002) comme la disponibilité et l'encouragement ou bien quand il a la souplesse d'esprit (Correa Molina, 2008), la sensibilité et la capacité d'adaptation (Altarugio et Souza Neto, 2019) pour comprendre les difficultés et fragilités de l'apprenti.

À partir de l'observation des plaintes des stagiaires sur leurs expériences partiellement satisfaisantes et insatisfaisantes, comme le manque d'intérêt des enseignants associés, par exemple, nous arrivons à la conclusion qu'une meilleure préparation de ces professionnels est fort souhaitable pour faire 
face à ce problème. Évidemment, cette préparation, qui présuppose le perfectionnement de quelques compétences spécifiques (Lamy, 2003; Chaliès et Durand, 2000; Altet, 2000), exige un programme de formation continue capable d'aider les agents concernés dans la formation des stagiaires à effectuer ce travail de manière plus professionnelle (Oliveira et Manrique, 2008). Il faut absolument corriger les problèmes montrés dans ce travail concernant la préparation de ces professionnels pour qu'ils comprennent le sens, l'importance et la complexité du processus des stages ainsi que leur rôle dans la formation des stagiaires (Altarugio et Souza Neto, 2019).

Une préparation préalable des formateurs, en plus de rendre possible le développement de compétences pour 1'accompagnement des stagiaires (Gervais, 2013; Gervais et Lepage, 2000; Portelance et al., 2008), peut favoriser l'importante articulation entre l'école et l'université. L'échange de savoirs entre les deux institutions réglerait des problèmes comme ceux dont il est question dans le présent travail, concernant l'incompatibilité d'objectifs et d'opinions et l'effort unilatéral, fatigant et inutile.

Au Brésil, l'encouragement à la préparation des enseignants associés et à l'articulation entre l'université et l'école, à peine suggéré dans les directives légales, représente le défi majeur des stages. À présent, ces défis sont envisagés isolément et de manière particularisée, sans aucun règlement formel. Pour composer avec ces défis, il faudrait les considérer dans le contexte d'une politique institutionnalisée (Behrend, Cousin et Schmidt, 2019; Portelance et al., 2008) de formation initiale et continue d'enseignants, si l'on veut que les superviseurs travaillent de manière efficace et collaborative dans le processus du stage.

Même si les stagiaires envisagent la participation au quotidien des écoles, l'échange d'expériences et l'encouragement à la réflexion, ayant lieu d'une manière dialogique dans la relation entre les étudiants et les superviseurs, comme une partie importante de leurs expériences réussies, il est essentiel que ces pratiques soient basées sur des savoirs scientifiques pour que ces futurs enseignants développent les compétences requises pour leur métier. Nous croyons que les savoirs pratiques issus de l'observation de la réalité scolaire et de l'échange d'expériences font partie de la formation professionnelle (Tardif, 2010). Toutefois, l'analyse des situations éducatives et celle de l'agir professionnel faites par les superviseurs et les enseignants associés ne peuvent être ni aléatoires ni superficielles, mais doivent se fonder sur des choix conscients et être basées sur la théorie (Caron et Portelance, 2017); à notre avis une condition indispensable pour la formation de professeurs compétents.

\section{Conclusion}

Malgré la petite taille de l'échantillon, le fait que cette recherche soit limitée à un groupe spécifique et que la vision des stagiaires soit la seule considérée, nous croyons que les résultats de cette étude ont fourni des pistes des conditions favorables pour les stages, quelques-unes étant d'ailleurs déjà confirmées par des recherches importantes dans ce domaine. D'un autre côté, des aspects doivent encore être approfondis, surtout au Brésil. Il faut découvrir, par exemple, dans quelle mesure les problèmes dans la formation des superviseurs et le manque de préparation des écoles et de leurs gestionnaires influencent la qualité de la formation des stagiaires.

Les opinions des étudiants sur les stages révèlent que les expériences réussies sont possibles même si les conditions ne sont pas idéales. Dans ce sens, les conditions qui favorisent les bons résultats, comme l'accueil des écoles et les qualités personnelles et professionnelles des formateurs, se sont montrées 
importantes pour les stagiaires. Devant ce résultat, une question s'impose : comment se servir de ces éléments dans un programme de formation d'une façon moins aléatoire et moins dépendante des caractéristiques spécifiques des ambiances et des personnes pour assurer des conditions égalitaires à tous les stagiaires?

En même temps, nous avons repéré les défis les plus importants à surmonter, comme les problèmes d'articulation entre l'université et l'école. Même pour une jeune université comme la nôtre, nous constatons que ce processus est lent, progressif et limité. D'autre côté, nous savons qu'une partie de la solution du problème est l'investissement dans un travail plus collaboratif, intentionnel, systématique et continu, basé sur des savoirs pratiques et théoriques, soutenu par des politiques institutionnalisées pour contrer ce problème. Au Brésil, amplifier la conscience de l'ampleur de ces défis et l'importance d'encourager les recherches dans ce domaine sont des actions essentielles pour faire les changements nécessaires.

\section{Références}

Altarugio, M. H. et Souza Neto, S. (2019). A orientação de estágio supervisionado pelo professor universitário novato: desafios, qualidades e implicações para o processo de formação docente. Dans : S. A.S. Monteiro (dir.), Formação Docente, Princípios e Fundamentos v.2, (p.290-300). Ponta Grossa (PR) : Atena Editora. DOI 10.22533/ at.ed.69919300524

Altarugio, M.H. et Souza Neto, S. (2018). O papel do orientador e a formação do professor reflexivo no estágio supervisionado da área de ciências. Acta Scientiae, 21(4), 174-191. https://doi.org/10.17648/acta.scientiae.v21iss4id4894

Altet, M. (2000). L'analyse de pratiques : une démarche de formation professionnalisante? Recherche Eْ Formation, 35, 2534. https://doi.org/10.3406/refor.2000.1668.

Altet, M., Perrenoud, P. et Paquay, L. (2003). A profissionalização dos formadores de professores. São Paulo : Artmed Editora.

Aroeira, K. P. (2015, octobre). Estágio na licenciatura e possibilidades para o diálogo entre a universidade e escola. Communication présentée au XII Congresso Nacional de Educação EDUCERE, Curitiba, PR. Repéré le 2 mars 2021 à https://educere.bruc.com.br/arquivo/pdf2015/19641 8842.pdf

Bardin, L. (2008). Análise de Conteúdo. Lisboa : Edições 70.

Behrend, D. M., Cousin, C. S. et Schmidt, E. B. (2019). A relação interinstitucional entre escola e universidade no contexto dos Estágios Curriculares Supervisionados. Laplage em Revista, 5(1), 85-94. https://doi.org/10.24115/S2446-6220201951613

Brau-Antony, S., \& Mieusset, C. (2013). Accompagner les enseignants stagiaires: une activité sans véritables repères professionnels. Recherche et Formation, 72, 27-40. https://doi.org/10.4000/rechercheformation.2022

Carmo, M. C. do, et Polydoro, S. A. J. (2010). Integração ao Ensino Superior em um curso de Pedagogia. Psicologia Escolar e Educacional, 14(2), 211-220. https://doi.org/10.1590/S1413-85572010000200005

Caron, J. et Portelance, L. (2017). Perceptions de stagiaires quant au soutien des deux formateurs à l'articulation entre théorie et pratique. Formation et profession, 25(1), 34-49. http://dx.doi.org/10.18162/fp.2017.367

Chaliès, S. et Durand, M. (2000). L'utilité discutée du tutorat em formation initiale des enseignants. Recherche E̊ Formation, 35, 145-180. https://doi.org/10.3406/refor.2000.1678.

Correa Molina, E. (2008). Les superviseurs de stage : des qualités pour un role formateur. Dans : Correa Molina, E., Gervais, C. et Rittershaussen. S. (dir.), Vers une conceptualisation de la situation de stage : explorations internationales (p.205-219). Sherbrooke : Éditions du CRP. 
Correa Molina; E. et Gervais, C. (2004). L'argumentation pratique comme accompagment réflexif du stagiaire. Communication présentée au atelier Quelle formation d'adultes pour réaliser « l'éducation tout au long de la vie »? 7a. Biennale de L'education et de la formation. Repéré le 2 mars 2021 à http://www.inrp.fr/biennale/7biennale/Contrib/longue/7102.pdf

Cyrino, M. et Souza Neto, S. (2014). O estágio curricular supervisionado na experiência brasileira e internacional. Revista Educação em Questão, 48(34), 86-115. https://doi.org/10.21680/1981-1802.2014v48n34ID5731

Desbiens, J.-F., Borges, C., \& Spallanzani, C. (2009). Investir dans la formation des personnes enseignantes associées pour faire du stage en enseignement un instrument de développement professionnel. Éducation Et Francophonie, 37(1), 6. https://doi.org/10.7202/037650ar

Elliott, K. M. et Shin, D. (2010). Student Satisfaction: An alternative approach to assessing this important concept, Journal of Higher Education Policy and Management, 24 (2),197-209. doi: 10.1080/1360080022000013518

Gervais, C. (2005). Se former en formant les autres. Vie Pédagogique, 137, 45-48. Repéré le 2 mars 2021à http://numerique.banq.qc.ca/patrimoine/details/52327/20902?docref=few9NGWhcPta9edS-YicVQ

Gervais, C. (2013). A organização dos estágios e o acompanhamento do desenvolvimento profissional dos estagiários em Quebec. Cadernos de Educação, 46, 23-44. http://dx.doi.org/10.15210/caduc.v0i46.4171

Gervais, C. et Correa Molina, E. (2008). Pour communiquer son expérience : l'explicitation argumentée de savoirs d'esneignants associés et de stagiaires. Dans : Correa Molina, E., Gervais, C. et Rittershaussen. S. (dir.), Vers une conceptualisation de la situation de stage : explorations internationales. (p.191-204). Sherbrooke : Éditions du CRP.

Gervais, C. et Desrosiers, P. (2005). L'école, lieu de formation d'enseignants : questions et repères pour l'accompagnement de stagiaires. Québec : Presses de l'Université Laval.

Gervais, C. et Lepage, M. (2000). Transfert de la responsabilité de l'évaluation en stage du superviseur vers l'enseignant associé : un pas de plus vers la professionnalisation. Dans : Martin, D. et al (dir.), Recherche et pratiques de formation de maîtres: Vers une pratique réfléchie et argumentée. (p.113-128). Sherbrooke : Éditions du CRP.

Gil, A. C. (1999). Métodos e técnicas de pesquisa social (6éd). São Paulo : Atlas.

Lamy, M. (2003). Dispositivos de formação de formadores de professores: qual profissionalização? Dans : M. Altet, P. Perrenoud, et L. Paquay (dir.), A profissionalização dos formadores de professores. (p.41-43). São Paulo : Artmed.

Le Boterf, G. (2002). Développer la compétence des professionnels (4éd.). Paris : Éditions d’Organisation.

Linhares, P. C. A., Irineu, T. H. S., Silva, J. N., Figueiredo, J. P. et Sousa, T. P. (2014). A importância da escola, aluno, estágio supervisionado e todo o processo educacional na formação inicial do professor. Revista Terceiro Incluído, 4(2), 115-127. DOI : $10.5216 /$ teri.v4i2.35258

Lüdke, M. (2009). Universidade, escola de educação básica e o problema do estágio na formação de professores. Formação Docente - Revista Brasileira de Pesquisa sobre Formação de Professores, 1(1), 95-108. Repéré le 2 mars 2021 à https://revformacaodocente.com.br/index.php/rbpfp/article/view/7

Malhotra, N. (2006). Pesquisa de marketing : uma orientação aplicada (4éd). Porto Alegre : Bookman.

Marconi, M.A. et Lakatos, E.M. (1990). Técnicas de pesquisa (2éd) S. Paulo: Atlas.

Martins, A. F. P. (2009). Estágio supervisionado em física: o pulso ainda pulsa... Revista Brasileira de Ensino de Física, 31(3), 3402.1-3402.7. http://dx.doi.org/10.1590/S1806-11172009000300006

Mazieiro, A. R. et Carvalho, D. G. (2012). A contribuição do supervisor de estágio na formação dos estagiários. Acta Scientiae, 14(1), 63-75. Repéré le 2 mars 2021 à http://www.periodicos.ulbra.br/index.php/acta/article/view/212

Milanesi, I. (2012). Estágio supervisionado: concepções e práticas em ambientes escolares. Educar em Revista, 46, 209-227 https://doi.org/10.1590/S0104-40602012000400015

Ministério da Educação (MEC) - Brazil (1996). Lei de Diretrizes e Bases da Educação Nacional (no 9394). Brasília, DF. Repéré le 20 février 2021 à http://www.planalto.gov.br/ccivil 03/Leis/L9394.htm

Ministério da Educação (MEC) - Brazil (2001a). Parecer CNE/CP (nº 009, de 8 de maio de 2001), Brasília, DF. Repéré le 20 février 2021 à http://portal.mec.gov.br/cne/arquivos/pdf/009.pdf 
Ministério da Educação (MEC) - Brazil (2001b). Parecer CNE/CP (n 28, de 2 de outubro de 2001). Brasília, DF. Repéré le 20 février 2021 à http://portal.mec.gov.br/cne/arquivos/pdf/028.pdf

Nico, J. B. (2000). O conforto acadêmico do(a) caloiro(a). Dans : Soares, A. P. et al. (dir). Transição para o ensino superior.(p. 161-166). Braga, Portugal : Reitoria da Universidade do Minho.

Oliveira, I. M. et Manrique, A. L. (2008, octobre) Um estudo sobre o estágio supervisionado em cursos de licenciatura em Matemática. Communication présentée au VIII Congresso Nacional de Educação EDUCERE, Curitiba, PR. Repéré le 2 mars 2021 à http://www.pucpr.br/eventos/educere/educere2008/anais/pdf/697 530.pdf

Pimenta, S. G. et Lima, M. S. L. (2004). Estágio e Docência. São Paulo : Cortez.

Portelance, L., Gervais, C., Lessard, M. et Beaulieu, P. et collaborateurs. (2008). La formation des enseignants associés et des superviseurs universitaires. Rapport de recherche. Cadre de référence. Repéré le 2 mars 2021 à https://www.usherbrooke. ca/education/fileadmin/sites/education/documents/Intranet/Documents officiels/Rapport Cadre reference 2009.pdf

Portelance, L.; Van Nieuwenhoven, C. (2010). Fondements théoriques et méthodologies des conduites de recherche portant sur la formation professionnelle en alternance. Éducation et francophonie, 38(2), 1-5. http://dx.doi.org/10.7202/ $1002159 \mathrm{ar}$

Rodrigues, M. A. (2013). Quatro diferentes visões sobre o estágio supervisionado. Revista Brasileira de Educação, 18(55). https://doi.org/10.1590/S1413-24782013000400011

Scalabrin, I. C. et Molinari, A. M. C. (2013) A importância da prática de estágio supervisionado nas licenciaturas. Revista Unar, 7(1). Repéré le 20 février 2021 à http://revistaunar.com.br/cientifica/documentos/vol7 n1 2013/3 a importancia da p ratica estagio.pdf

Schleich, A. L. R.; Polydoro, S.A. J. et Santos, A. A. A. (2006). Escala de satisfação com a experiência acadêmica de estudantes do ensino superior. Avaliação Psicológica, 5(1), 11-20. Repéré le 20 février 2021 à http://pepsic.bvsalud.org/ scielo.php?script=sci arttext\&pid=S1677-04712006000100003\&lng=pt\&tlng=pt.

Souza Neto, S., Sarti, F. M. et Benites, L. C. (2016). Entre o ofício de aluno e o habitus de professor : os desafios do estágio supervisionado no processo de iniciação à docência. Movimento, Porto Alegre, 22(1), 311-324. https://doi. org/10.22456/1982-8918.49700

Rozelle, J. J., et Wilson, S. M. (2012). Opening the black box of field experiences: How cooperating teachers'beliefs and practices shape student teachers' beliefs and practices. Teaching and Teacher Education, 28(8), 1196-1205. https://doi. org/10.1016/j.tate.2012.07.008

Tardif, M. (2010). Saberes docentes e formação profissional (14éd). Petrópolis: Vozes.

\section{Pour citer cet article}

Leite da Silva, A. et Altarugio, M.H. (2021), Les conditions favorables à la réussite d'un stage selon les stagiaires en formation au Brésil. Formation et profession, 29(3), 1-13. http://dx.doi.org/10.18162/fp.2021.606 